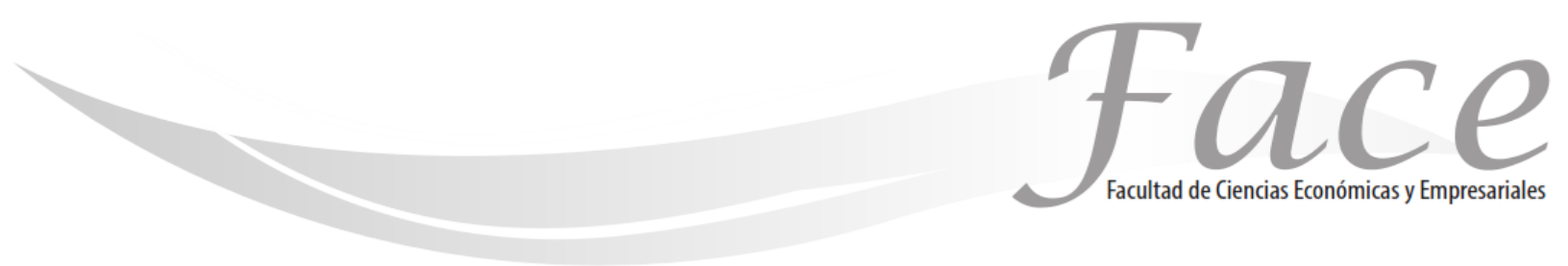

ISSN Impreso: 1794-9920

ISSN Electrónico: 2500-9338

Volumen 17-N²

Año 2017

Págs. 196 - 205

\title{
EVALUACIÓN DE LA SATISFACCIÓN DEL CLIENTE EN EL SECTOR FINANCIERO DEL MUNICIPIO DE PAMPLONA - COLOMBIA
}

\author{
Gustavo Enrique Bohórquez * \\ Enlace ORCID: https://orcid.org/0000-0003-1215-4587 \\ Jose Sergio Fernández ** \\ Enlace ORCID: https:// orcid.org/0000-0002-2302-4115
}

Fecha de Recepción: 15 de junio 2017

Fecha de Aprobación: 10 de Julio 2017

\section{Resumen:}

El sector financiero requiere elevados niveles de eficiencia y calidad de servicio para lograr su desarrollo; la presente investigación se planteó como objetivo Generar propuestas de mejora para aumentar el nivel de satisfacción de los clientes a través de la caracterización y análisis de la dinámica de atención al cliente en el sector financiero del municipio Pamplona. Se desarrolló un estudio descriptivo con base en un diseño de campo; se evalúo la calidad de servicio percibida por los clientes internos y externos utilizando los elementos teóricos del Modelo Servqual. Los resultados obtenidos permitieron identificar las brechas existentes para cada una de las dimensiones, a partir de estos elementos se desarrollaron propuestas para lograr la mejora en la calidad percibida. Además de las acciones generales para las instituciones del sector se recomienda el estudio detallado para ajustar los lineamientos a cada organización particular.

Palabras clave: Sector Financiero, Modelo Servqual, Calidad de servicio. Financial Sector, Model Servqual, Quality of service, statistics.

*Docente Catedrático Instituto Superior de Educación Rural, ISER, Ingeniero industrial, Especialista en indicadores de gestión, Magister en gerencia de empresas. Correo: ingustavo79@gmail.com

** Docente Universidad Nacional del Chaco Austral, Argentina, Ingeniero, Esp. en Ingeniería Gerencial, MSc. en Administración de Negocios, PhD. en Ingeniería. Correo: sergiof@uncaus.edu.ar. 


\title{
EVALUATION OF CUSTOMER SATISFACTION IN THE FINANCIAL SECTOR OF THE MUNICIPALITY OF PAMPLONA - COLOMBIA.
}

\begin{abstract}
:
The financial sector requires high levels of efficiency and quality of service to achieve its development; The present research was aimed at generating improvement proposals to increase the level of customer satisfaction through the characterization and analysis of the dynamics of customer service in the financial sector of the Pamplona municipality. A descriptive study was developed based on a field design; The quality of service perceived by internal and external customers is evaluated using the theoretical elements of the Servqual Model. The results obtained allowed to identify the existing gaps for each of the dimensions, from these elements proposals were developed to achieve the improvement in perceived quality. In addition to the general actions for the institutions of the sector we recommend the detailed study to adjust the guidelines to each particular organization.
\end{abstract}

Keywords: Financial Sector, Model Servqual, Quality of service.

\section{AVALIAÇÃO DA SATISFAÇÃO DO CLIENTE NO SETOR FINANCEIRO DO MUNICÍPIO DE PAMPLONA - COLÔMBIA}

Resumo:

O setor financeiro exige altos níveis de eficiência e qualidade de serviço para alcançar seu desenvolvimento; A presente investigação foi proposta como um objetivo Gerar propostas de melhoria para aumentar o nível de satisfação do cliente através da caracterização e análise da dinâmica de atendimento ao cliente no setor financeiro de Pamplona. Um estudo descritivo foi desenvolvido com base em um projeto de campo; a qualidade do serviço percebido pelos clientes internos e externos foi avaliada utilizando os elementos teóricos do Modelo Servqual. Os resultados obtidos permitiram identificar as lacunas existentes para cada uma das dimensões, a partir desses elementos foram desenvolvidas propostas para alcançar a melhoria na qualidade percebida. Além das ações gerais das instituições do setor, recomenda-se um estudo detalhado para ajustar as diretrizes a cada organização em particular.

Palavras-chave: Setor Financeiro, Modelo Servqual, Qualidade de serviço. Setor Financeiro, Modelo Servqual, Qualidade de serviço, estatística. 


\section{INTRODUCCIÓN:}

En Colombia la concentración de la banca se ha dado en el mercado corporativo empresarial y sector público, pero este mercado, ha sido reevaluado debido a la crisis financiera internacional. Solo bancos que tenían una gran diversificación y base de clientes robusta fueron los que pudieron superar la crisis, además, si el banco cuenta con una base de clientes personales muy amplia la autoridad de regulación deberá ir en su rescate.

En Pamplona, Norte de Santander, se puede evidenciar el comportamiento del mercado financiero nacional debido a la presencia de sucursales de los grandes grupos financieros que concentran el grueso de la actividad y sucursales de cooperativas financieras y pequeñas entidades dedicadas al microcrédito. La Banca Colombiana se enfrenta a una gran competencia; en este contexto Guiliany y otros (2017), plantea que se requieren nuevas organizaciones basadas en redes y equipos de trabajo, que usen tecnologías, procesos flexibles, los cuales deben tener en cuenta a los clientes.

La investigación desarrollada, permitió identificar lineamientos de actuación para el sector financiero del Municipio Pamplona, los resultados de este estudio se muestran en este informe. En el Capítulo I se describe el problema y las preguntas de investigación, así como la descripción de los aportes que justifican esta investigación. En el ítem 2 se describen los antecedentes y las bases teóricas que permitieron definir como desarrollar el proceso investigativo, en el ítem 3 se mencionan aspectos metodológicos, en el ítem 4 los resultados del análisis de los datos. En el Capítulos V se describen las acciones propuestas y en el capítulo $\mathrm{VI}$ las conclusiones $\mathrm{y}$ recomendaciones.

\section{MARCO TEÓRICO:}

La prestación de un servicio con baja calidad implica pérdida de la clientela, genera incremento en los costos, reducción en los beneficios por los errores cometidos, deterioro del personal por la desmotivación que producen las quejas de los clientes y el mal funcionamiento del servicio.

Con base en los objetivos propuestos para esta investigación, se revisaran como antecedentes, investigaciones previas a nivel nacional e internacional relacionadas con calidad de servicio y satisfacción del cliente en diversos sectores, así como estudios de aplicación de la teoría de colas como herramienta para la mejora en los servicios.
Antecedentes Internacionales:

A nivel internacional existe una tesis doctoral dirigida al sector financiero desarrollada por Merino, J (2003) en la Universidad Complutense de Madrid, Facultad de Ciencias Económicas y Empresariales departamento de comercialización e investigación de Mercados intitulada "La Calidad de Servicio Bancario: Entre la Fidelidad y la Ruptura". Los objetivos de esta investigación fueron primero medir la calidad de servicio en las entidades financieras; en segundo lugar, analizar los aspectos que componen la calidad de servicio de las entidades financieras y la relación existente entre calidad de servicio, satisfacción y fidelidad del cliente.

\section{Antecedentes Nacionales:}

A nivel nacional se consideran dos antecedentes importantes que se toman como referencia para desarrollar la presente investigación. En primera instancia, la tesis de Maestría desarrollada por Ibarra (2004), en el Instituto Politécnico Nacional - Escuela Superior de Turismo de la ciudad de México, intitulada "Calidad en el Servicio al Cliente como Estrategia Competitiva en los dos Principales Hoteles de Cúcuta Colombia", cuyo objetivo fue evaluar la calidad del servicio como estrategia competitiva entre los dos principales Hoteles de la Ciudad de Cúcuta-Colombia con el fin de desarrollar un plan de mejoramiento que permita aumentar la Satisfacción del Cliente. Por otro lado, Arias \& Perez, R. (2015), determina que los mercados globales cada vez más exigentes son algunas de las características de un mundo que presiona altamente cada día más en las organizaciones. Desde el contexto económico Laguado, y otros (2013), identifican desde el contexto local la influencia de las trasferencias de la nación y espacialmente su efecto en el sector financiero y la influencia por parte de la Universidad de Pamplona. El anterior planteamiento permite fortalece con los procesos de producción, con las relaciones intermunicipales, con el mejoramiento de los procesos de valoración, recuperación y fortalecimiento de las identidades para la construcción de territorialidad, (Mosquera \& Reyes2014).

\section{Bases Teóricas:}

La teoría necesaria para el desarrollo de esta investigación se obtuvo de fuentes bibliográficas y documentos en línea 
relacionados con: Filosofía de la Calidad, Calidad de Servicio, evaluación de la satisfacción del cliente y teoría de colas.

Calidad:

Según lo planteado por Stoner y Finch (1996)la calidad: "Se refiere a lograr productos y servicios cada vez mejores y a precios más competitivos." La importancia de la calidad en el servicio puede resumirse de la siguiente manera: servicios.

- Los clientes exigen cada vez más y mejores

- Los clientes están cada vez más conscientes de sus necesidades y deseos.

consumidores finales.

- El servicio fomenta la lealtad de los

- Un buen servicio es en realidad una garantía de supervivencia.

La satisfacción de los clientes se alcanza mediante la búsqueda de la excelencia en los procesos en este sentido, Horovitz (1991), define la calidad como el nivel de excelencia que una empresa decide alcanzar para satisfacer a su clientela clave; con este fin privilegia los siguientes factores asociados a este concepto: Necesidades del Cliente, Expectativas del cliente, Gestión y manejo de las expectativas del cliente

\section{Calidad de Servicio}

Un servicio de calidad debe ajustarse a las especificaciones del cliente, el cliente percibe lo que ocurre basándose en sus expectativas de servicio. Un buen sistema de calidad del servicio se puede constituir en el diferenciador de los servicios que se ofrecen, los cuales deben distinguirse por los niveles de satisfacción de los clientes y por el sistema que soporta esos índices de desempeño que se informan con puntualidad, validez y pertinencia a los clientes.

\section{Satisfacción del cliente:}

La satisfacción del cliente es un término propio del marketing que hace referencia al nivel de conformidad que tiene un cliente respecto a un producto que ha comprado 0 un servicio que ha recibido, cuándo éste ha cumplido o sobrepasado sus expectativas. Dado que la satisfacción del cliente influye en su comportamiento, es una meta muy valiosa para toda organización, si las expectativas del cliente son bajas o si el cliente tiene acceso limitado a cualquiera de los servicios, puede ser que esté satisfecho con recibir servicios relativamente deficientes
Parasuraman, Zeithalm y Berry (1985) identificaron un modelo constituido por dimensiones o factores. Las definiciones de las cinco nuevas dimensiones o factores son las siguientes:

1. Elementos Tangibles. Apariencia de las instalaciones físicas, equipos, personal y materiales de comunicación.

2. Fiabilidad o confiabilidad. Habilidad para realizar el servicio prometido de forma fiable y cuidadosa.

3. Capacidad de respuesta (Diligencia). Disposición y voluntad para ayudar a los usuarios y proporcionar un servicio rápido.

4. Seguridad (garantía). Conocimiento y atención mostrados por los empleados y sus habilidades para inspirar credibilidad y confianza.

5. Empatía. Atención individualizada que ofrecen las empresas a sus consumidores.

\section{METODOLOGÍA:}

Nivel de la Investigación

La clasificación del tipo de estudio, es de gran importancia, pues de él depende la estrategia de investigación. Sampieri y Collado (2006), plantean que para resolver un problema utilizando el abordaje científico, es conveniente conocer en forma detallada las posibles modalidades de investigación que se pueden aplicar. Según Sampieri y Collado (2006) la investigación no experimental se realiza sin manipular deliberadamente las variables, sólo se observan los fenómenos tal como se dan en su contexto natural, para después analizarlos.

Esta investigación está orientada en el análisis del nivel de satisfacción del cliente en las entidades bancarias existentes en la ciudad de Pamplona, adicionalmente se propone ejecutar un estudio de Teoría de Colas para identificar las dificultades en el proceso de atención y proponer acciones para mejorar la eficiencia en la operación del sistema de servicio, la aplicación de esta teoría permite encontrar un balance adecuado entre el costo de servicio y la cantidad de espera. 
Esta investigación está enmarcada en el paradigma cuantitativo y se apoya en una investigación descriptiva de acuerdo con lo expuesto por Arias (2006):

La investigación descriptiva consiste en la caracterización de un hecho, fenómeno, individuo o grupo, con el fin de establecer su estructura o comportamiento. Los resultados de este tipo de investigación se ubican en un nivel intermedio en cuanto a la profundidad de los conocimientos se refiere. (p. 24).

\section{DISEÑO DE LA INVESTIGACIÓN}

La investigación está enmarcada dentro de un diseño de campo, ya que de acuerdo con Balestrini (1997), señala que "la investigación de campo permite observar los datos directamente de la realidad de los objetos de estudio, en un ambiente cotidiano para posteriormente analizar e interpretar los resultados de sus indagaciones" (p. 15).

\section{POBLACIÓN}

De acuerdo con lo expresado por Balestrini (1997), la población o universo de estudio está referida a "...cualquier conjunto de elementos de los cuales pretendemos indagar y conocer sus características, o una de ellas, y para el cual serán válidas las conclusiones obtenidas en la investigación." (p. 122). El estudio se realizará sobre las entidades financieras del municipio Pamplona, con el propósito de lograr una visión integral del problema objeto de estudio se manejaran dos poblaciones de informantes.

Población 1: conformada por los clientes y usuarios de las entidades financieras del Municipio Pamplona, en la Tabla 1 se observan los datos disponibles, se tienen un total de 38830 clientes distribuidos en las 10 entidades financieras.

Población 2: corresponde al número de trabajadores de cada entidad bancaria, en la tabla 2 se describe el número de personas correspondientes a cada organización.
Tabla 1. Nro.de clientes por entidad financiera del Municipio Pamplona

\begin{tabular}{c|c}
\hline \multicolumn{2}{c}{ ENTIDADES FINANCIERAS } \\
\hline BANCO O COOPERATIVA & $\mathrm{N}^{\circ}$ de Clientes \\
\hline BANCOLOMBIA & 3.820 \\
\hline BANCO DE BOGOTA & 7.120 \\
\hline BBVA & 7.050 \\
\hline BANCOOMEVA & 560 \\
\hline BANCO POPULAR & 5.680 \\
\hline DAVIVIENDA & 550 \\
\hline BANCO AGRARIO & 3.780 \\
\hline BANCAMIA & 3510 \\
\hline COOMULTRASAN & 2.750 \\
\hline \hline
\end{tabular}

Fuente: Cámara de Comercio de Pamplona

Tabla 2 Empleados por entidad financiera

\begin{tabular}{lccc}
\hline \multicolumn{4}{c}{ EMPLEADOS POR ENTIDAD FINANCIERA } \\
\hline $\begin{array}{c}\text { BANCO 0 } \\
\text { COOPERATIVA }\end{array}$ & $\begin{array}{c}\text { NÚMERO DE } \\
\text { EMPLEADOS }\end{array}$ & $\begin{array}{c}\text { BANCO O } \\
\text { COOPERATIVA }\end{array}$ & $\begin{array}{c}\text { NÚMERO DE } \\
\text { EMPLEADOS }\end{array}$ \\
\hline BANCOLOMBIA & 11 & DAVIVIENDA & 7 \\
\hline $\begin{array}{l}\text { BANCO DE } \\
\text { BOGOTA }\end{array}$ & 15 & BANCO AGRARIO & 11 \\
\hline BBVA & 13 & BANCAMIA & 12 \\
\hline BANCOOMEVA & 7 & COOMULTRASAN & 8 \\
\hline BANCO POPULAR & 14 & $\begin{array}{l}\text { BANCO DE LA } \\
\text { MUJER }\end{array}$ \\
\hline TOTAL & & 113 & 15 \\
\hline
\end{tabular}




\section{MUESTRA}

La muestra es un subconjunto de elementos que pertenecen a la población de estudio, para las dos poblaciones de esta investigación se realizará un muestreo aleatorio estratificado con el fin de que todas las personas en la muestra tengan la misma posibilidad de ser seleccionadas y se determine el nivel de satisfacción del cliente mediante la evaluación del servicio prestado en el sector financiero del Municipio de Pamplona. En la Tabla 3 se muestran los resultados del cálculo de la muestra.

Según Scheaffer, Mendenhall y Ott(1990). La fórmula de muestreo aleatorio simple es:

Donde:

$n=\frac{p \times q}{\frac{e^{2}}{Z^{2}}+\frac{p \times q}{N}}$

$\mathbf{Z}=$ Grado de confianza. Para esta investigación, el valor de $Z$ corresponde a $3 \sigma$; esto quiere decir, que se trabajará con un valor de 1,96.

$\mathbf{e}=$ Margen de error: es un estadístico que expresa el error de muestreo aleatorio aceptado el resultado de una encuesta. Para este proyecto el margen utilizado es del $5 \%$.

$\mathbf{N}=$ Tamaño de la población, en la población 1 está determinada por los clientes que adquieren servicios de las entidades bancarias, corresponde a 38.830 personas; en la población 2 el total de empleados es 113 personas.

$p=$ probabilidad de éxito, será igual 0,5 .

$q=$ probabilidad de fracaso, será igual 0,5 .
Tabla 3. Tamaño de la muestra por estrato para cada población

\begin{tabular}{c|c|c|c|c}
\hline $\begin{array}{c}\text { TAMAÑO DE LA } \\
\text { MUESTRA }\end{array}$ & $\begin{array}{c}\text { Muestra } \\
\text { Clientes }\end{array}$ & $\begin{array}{c}\text { Muestra } \\
\text { empleados }\end{array}$ \\
\hline $\begin{array}{c}\text { BANCO O } \\
\text { COOPERATIVA }\end{array}$ & $\begin{array}{c}\mathrm{N}^{\circ} \text { de } \\
\text { Clientes }\end{array}$ & & $\begin{array}{c}\text { No de } \\
\text { empleados }\end{array}$ & \\
\hline BANCOLOMBIA & 3.820 & 38 & 11 & 9 \\
\hline $\begin{array}{c}\text { BANCO DE } \\
\text { BOGOTA }\end{array}$ & 7.120 & 70 & 15 & 12 \\
\hline BBVA & 7.050 & 69 & 13 & 9 \\
\hline BANCOOMEVA & 560 & 6 & 7 & 5 \\
\hline BANCO POPULAR & 5.680 & 56 & 14 & 11 \\
\hline DAVIVIENDA & 550 & 5 & 7 & 5 \\
\hline BANCO AGRARIO & 3.780 & 37 & 11 & 9 \\
\hline BANCAMIA & 3510 & 34 & 12 & 9 \\
\hline COOMULTRASAN & 2.750 & 27 & 8 & 6 \\
\hline BANCO DE LA \\
MUJER & 4.010 & 39 & 15 & 12 \\
\hline TOTAL & 38.830 & 381 & 113 & 87 \\
\hline Clientes & & & \\
\hline
\end{tabular}

Técnicas de Recolección de Datos

Según Sampieri y Collado (2006) una vez seleccionado el diseño de la investigación y determinada la muestra adecuada, la siguiente etapa consiste en la recolección de los datos pertinentes relacionados con las variables e indicadores seleccionados para la investigación. En esta investigación se aplicará un cuestionario a los usuarios de servicios financieros en la ciudad de Pamplona y otro a los empleados de las instituciones financieras; la aplicación de estos instrumentos se realizará mediante la técnica de la encuesta 

en los servicios ofrecidos por las entidades
financieras.

Análisis E Interpretación de Resultados:

En este capítulo se exponen los resultados obtenidos de los cuestionarios aplicados a los Gerentes, personal Administrativo, clientes y usuarios de las entidades financieras del municipio de Pamplona, Norte de Santander, los cuales permitirán dar respuesta a cada una de las inquietudes que se presentan en la investigación. Los resultados se presentan de acuerdo con los objetivos de investigación, agrupados según las variables e indicadores seleccionados.

Objetivo 1: Identificar las variables que perciben y valoran los clientes internos como factores de calidad en los servicios ofrecidos por las entidades financieras.

Para desarrollar este objetivo se seleccionó como variable la calidad de servicio, definida en cinco dimensiones: elementos tangibles, fiabilidad o confiabilidad, capacidad de respuesta, seguridad y empatía.

Objetivo 2: Medir el nivel de satisfacción de los clientes externos del sector bancario en la ciudad de Pamplona

Para desarrollar este objetivo se seleccionó como variable el nivel de satisfacción de los clientes, definida en cinco dimensiones: elementos tangibles, fiabilidad 0 confiabilidad, capacidad de respuesta, seguridad y empatía.

\section{RESULTADOS Y DISCUSIÓN:}

En este ítem se exponen los resultados obtenidos de los cuestionarios aplicados a los Gerentes, personal Administrativo, clientes y usuarios de las entidades financieras del municipio de Pamplona, Norte de Santander, los cuales permitirán dar respuesta a cada una de las inquietudes que se presentan en la investigación. Los resultados se presentan de acuerdo con los objetivos de investigación, agrupados según las variables e indicadores seleccionados.

Objetivo 1: Identificar las variables que perciben y valoran los clientes internos como factores de calidad
Para desarrollar este objetivo se seleccionó como variable la calidad de servicio, definida en cinco dimensiones: elementos tangibles, fiabilidad o confiabilidad, capacidad de respuesta, seguridad y empatía.

\section{Objetivo 2: Medir el nivel de satisfacción de los clientes externos del sector bancario en la ciudad de Pamplona}

Para desarrollar este objetivo se seleccionó como variable el nivel de satisfacción de los clientes, definida en cinco dimensiones: elementos tangibles, fiabilidad 0 confiabilidad, capacidad de respuesta, seguridad y empatía.

\section{Factores de calidad}

Sector financiero del municipio pamplona

Tabla 4 Resultados objetivo 1

\begin{tabular}{|c|c|c|}
\hline Dimensiones & Indicador & Resultado \\
\hline \multirow{3}{*}{$\begin{array}{l}\text { Elementos } \\
\text { tangibles }\end{array}$} & $\begin{array}{l}\text { Organización de } \\
\text { los servidores o } \\
\text { canales de } \\
\text { servicio }\end{array}$ & Favorable \\
\hline & $\begin{array}{l}\text { Grado de } \\
\text { utilización de los } \\
\text { equipos de } \\
\text { computación. }\end{array}$ & Favorable \\
\hline & $\begin{array}{l}\text { Nivel de } \\
\text { comodidad } \\
\text { percibido por los } \\
\text { funcionarios }\end{array}$ & Favorable \\
\hline \multirow{3}{*}{$\begin{array}{l}\text { Fiabilidad o } \\
\text { confiabilidad }\end{array}$} & $\begin{array}{l}\text { Grado de } \\
\text { satisfacción de } \\
\text { los usuarios y } \\
\text { clientes. }\end{array}$ & Favorable \\
\hline & $\begin{array}{l}\text { Nivel de } \\
\text { capacitación de } \\
\text { los funcionario }\end{array}$ & Favorable \\
\hline & $\begin{array}{l}\text { Volumen de } \\
\text { solicitudes de } \\
\text { alta complejidad }\end{array}$ & $\begin{array}{c}\text { Alto } \\
\text { volumen }\end{array}$ \\
\hline $\begin{array}{l}\text { Capacidad de } \\
\text { respuesta }\end{array}$ & $\begin{array}{l}\text { Volumen de } \\
\text { solicitudes } \\
\text { internas }\end{array}$ & $\begin{array}{c}\text { Alto } \\
\text { volumen }\end{array}$ \\
\hline
\end{tabular}




\begin{tabular}{|c|c|c|}
\hline & $\begin{array}{l}\text { atendidas a } \\
\text { tiempo }\end{array}$ & \\
\hline & $\begin{array}{l}\text { Tiempo de } \\
\text { atención para } \\
\text { una solicitud } \\
\text { externa. }\end{array}$ & $\begin{array}{l}\text { Tiempo } \\
\text { de } \\
\text { servicio } \\
\text { elevado }\end{array}$ \\
\hline \multirow{3}{*}{ Seguridad } & $\begin{array}{l}\text { Grado de } \\
\text { desenvolvimiento } \\
\text { en la atención } \\
\text { de clientes y } \\
\text { usuarios }\end{array}$ & $\begin{array}{c}\text { Alta } \\
\text { demanda }\end{array}$ \\
\hline & $\begin{array}{l}\text { Actitud de } \\
\text { servicio }\end{array}$ & Amable \\
\hline & $\begin{array}{l}\text { Nivel académico } \\
\text { del personal. }\end{array}$ & Nivel alto \\
\hline Empatía & $\begin{array}{l}\text { Capacidad de } \\
\text { atender con } \\
\text { amabilidad }\end{array}$ & $\begin{array}{c}\text { Alta } \\
\text { capacidad }\end{array}$ \\
\hline
\end{tabular}

\section{PROPUESTA}

Acciones para aumentar el nivel de satisfacción de los clientes en el sector financiero del Municipio Pamplona.

\section{Exposición de Motivos}

El dinamismo y las condiciones de mercado en el sector financiero obligan a las empresas que lo conforman a mantenerse actualizadas y ofrecer servicios rápidos y oportunos. La evolución de la banca está muy relacionada con el desarrollo de las tecnologías de información, este hecho genera cambios en las expectativas de los clientes y obliga que cada vez debe aumentarse el nivel de calidad que ofrecen las empresas para ajustarse a crecientes expectativas de los clientes y usuarios. Los resultados obtenidos sirven como guías generales respecto a las debilidades y aspectos a mejorar por cada institución particular, por lo tanto, es necesario que cada una de ellas efectúe la revisión para ajustar las acciones propuestas a su caso particular. En este sentido, a partir de los resultados de la investigación se identificaron las acciones necesarias para lograr incrementar el nivel de calidad y el nivel de satisfacción de los clientes, se formularon acciones generales que posteriormente sirvan para orientar las acciones particulares para cada empresa.

Con base en la identificación de los factores de calidad y la percepción de los clientes internos y externos respecto al nivel de calidad ofrecida por las instituciones del sector financiero se consolidó la información y se construyó el resumen presentado en la Tabla a continuación, donde se describe el conjunto de acciones propuesto para el sector formuladas con base en los resultados previos.

Objetivo 1: Identificar las variables que perciben y valoran los clientes internos como factores de calidad en los servicios ofrecidos por las entidades financieras.

La calidad de servicio evaluada mediante la aplicación del modelo SERVQUAL, enfocada en la perspectiva del cliente interno permitió identificar brechas de calidad en cuatro indicadores.

El primero indicador está asociado con la Dimensión Fiabilidad corresponde al volumen de solicitudes de alta complejidad que se reciben y son procesadas por el personal de la entidad bancaria, el volumen elevado de transacciones genera retrasos e impacta el tiempo disponible para atender a los clientes externos. El segundo está asociado con la Dimensión Capacidad de respuesta, se relaciona con el número elevado de requerimientos internos y el consumo de tiempo para su manejo, afectando también a los clientes externos. Estos indicadores requieren acciones en el mediano plazo.

Se detectaron también dos indicadores que requieren atención inmediata:

El primero está asociado con la Dimensión capacidad de respuesta y se refiere al tiempo de atención a los usuarios externos, se detectó que es elevado y junto con el gran volumen de personas que acuden a las entidades financieras genera inconformidad. El segundo indicador se refiere a la elevada demanda de atención por parte de los usuarios lo cual afecta el desenvolvimiento de los empleados, este indicador está asociado con la Dimensión Seguridad.

\section{Objetivo 2: Medir el nivel de satisfacción de los clientes externos del sector bancario en la ciudad de Pamplona}

Una vez aplicado el proceso de evaluación por medio de las cinco dimensiones del Modelo SERVQUAL desde la perspectiva de los clientes externos, se detectó que existen deficiencias en todas las dimensiones, algunas requieren 


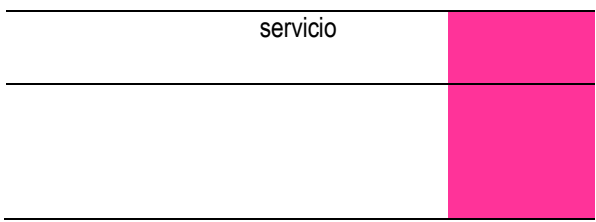

atención inmediata y otras pueden ser atendidas en el mediano plazo. Tal como se muestra en la Tabla:

Tabla 5 Resultados objetivo 2

\begin{tabular}{|c|c|c|}
\hline Dimensiones & Indicador & Resultado \\
\hline \multirow{3}{*}{$\begin{array}{l}\text { Elementos } \\
\text { Tangibles }\end{array}$} & $\begin{array}{l}\text { Grado de } \\
\text { adecuación en la } \\
\text { infraestructura }\end{array}$ & $\begin{array}{l}\text { Mediano } \\
\text { plazo }\end{array}$ \\
\hline & $\begin{array}{l}\text { Nivel de } \\
\text { disponibilidad de } \\
\text { los recursos }\end{array}$ & Inmediato \\
\hline & $\begin{array}{lr}\text { Número } & \text { de } \\
\text { servidores } & 0 \\
\text { canales } & \text { de } \\
\text { servicio } & \end{array}$ & Inmediato \\
\hline \multirow{3}{*}{ Confiabilidad } & $\begin{array}{l}\text { Eficacia en el } \\
\text { servicio }\end{array}$ & $\begin{array}{l}\text { Mediano } \\
\text { plazo }\end{array}$ \\
\hline & $\begin{array}{l}\text { Nivel de } \\
\text { importancia que } \\
\text { se percibe hacia } \\
\text { las solicitudes }\end{array}$ & Inmediato \\
\hline & $\begin{array}{ll}\text { Grado } & \text { de } \\
\text { cumplimiento en } \\
\text { la prestación del } \\
\text { servicio }\end{array}$ & Inmediato \\
\hline \multirow{4}{*}{$\begin{array}{l}\text { Capacidad de } \\
\text { respuesta }\end{array}$} & $\begin{array}{lr}\text { Tiempo } & \text { de } \\
\text { atención } & \text { a } \\
\text { clientes } & \end{array}$ & $\begin{array}{l}\text { Mediano } \\
\text { Plazo }\end{array}$ \\
\hline & $\begin{array}{lr}\text { Tiempo } & \text { de } \\
\text { atención } & \text { a } \\
\text { usuarios } & \end{array}$ & $\begin{array}{l}\text { Mediano } \\
\text { Plazo }\end{array}$ \\
\hline & $\begin{array}{l}\text { Tipo de servicio } \\
\text { como cliente }\end{array}$ & Favorable \\
\hline & $\begin{array}{l}\text { Tipo de servicio } \\
\text { como usuario }\end{array}$ & Favorable \\
\hline \multirow{3}{*}{ Seguridad } & $\begin{array}{l}\text { Grado de } \\
\text { desenvolvimiento } \\
\text { en la atención } \\
\text { de clientes y } \\
\text { usuarios }\end{array}$ & Inmediato \\
\hline & $\begin{array}{l}\text { Calificación del } \\
\text { servicio }\end{array}$ & Inmediato \\
\hline & $\begin{array}{l}\text { Calidad } \\
\text { servicio }\end{array}$ & Inmediato \\
\hline Empatía & Actitud & Inmediato \\
\hline
\end{tabular}

La investigación desarrollada permitió identificar los factores de calidad que perciben losclientes internos y externos en los procesos de atención de las instituciones que conforman el sector financiero del Municipio Pamplona. A partir de esta información y con base en el deber ser que propone la teoría es posible formular lineas de acción generales. Las acciones específicas que se ajustan a cada organización que opera en el sector estudiado requieren un estudio adicional que excede el alcance de este proyecto, sin embargo, a partir del plan general descrito en este capítulo las organizaciones puedenevaluar sus procesos y definir las actividades y tareas específicas que le permitirán el desarrollo de acciones particulares.

\section{REFERENCIAS:}

Arias, F. (2006). El proyecto de investigación. Guía para su elaboración. Caracas. Editorial Episteme.

Arias, M., \& Perez, R. (2015). EMPRENDIMIENTO: COMO COMENZAR UNA EMPRESA CON ÉXITO. FACE: Revista de la Facultad de Ciencias Económicas y Empresariales, 14, 175-183.

Balestrini, M. (1997). Cómo se Elabora el Proyecto de Investigación. Caracas: B.L Consultores.

Banco de la República. [Página web en línea]. Disponible en: www.banrep.gov.co

Berry,l.; Bennett,D. y Brown, C. (1990). Calidad de servicio: una ventaja estratégica para instituciones financieras. Editorial Diaz de Santos. Madrid. España.

Bierman H. Jr, Bonini C.P y Hausman W.H (2004). Análisis cuantitativo para la toma de decisiones. McGraw Hill. Interamericana Editores S.A. Madrid. España. 
Centro Latinoamericano para el desarrollo sostenible (2009). Informe Global de Competitividad 20092010 del Foro Económico Mundial. [Página web en línea]. Disponible en: http://conocimiento.incae.edu/ES/clacd/nuestrosproyectos/archivo-proyectos/proyectos-decompetitividad-clima-denegocios/WebsiteWEF/index.htm

Clavijo, S (2000). Hacia la multibanca en colombia: retos y retazos financieros. Disponible en: http://www.banrep.gov.co/docum/ftp/borra150.pdf

Colunga Dávila, C. (1995). La calidad en el servicio. Editorial Panorama. Méjico D.F.

Costa, J. (2002). Imagen Corporativa en el siglo XXI. Argentina. La Crujía.

Figueroa, E. (2008). Quien se llevo mi cliente?.Hispanic Business Consultant. California. U.S.A.

Gómez Jiménez, F. (2011). Aplicación de teoría de colas en una entidad financiera: herramienta para el mejoramiento de los procesos de atención al cliente. Revista Universidad EAFIT, [S.I.], v. 44, n. 150, p. 51-63, mar. 2011. ISSN 0120-341X. Disponible en: $<$ http://publicaciones.eafit.edu.co/index.php/revist a-universidad-eafit/article/view/154>. Fecha de acceso: 13 jul. 2015

Guiliany, J. E. G., Duran, S. E., \& Ronald, R. P. P. (2017). Políticas de gestión de talento humano para el desarrollo de competencias gerenciales en empresas metalmecánica. FACE: Revista de la Facultad de Ciencias Económicas y Empresariales, 17(2), 130-141.

Hayes, B. (1999). Como medir la satisfacción del cliente. Impresora Castillo Hermanos. México, D.F.

Horovitz, J. (1991). La calidad de servicio. Editorial McGraw Hill / Interamericana de España.

Ibarra Cáceres, T. (2004). La calidad en el servicio al cliente como estrategia competitiva en los dos principales hoteles de la ciudad de Cúcuta Colombia [Tesis de Maestría]. Instituto Politécnico Nacional. Méjico. Disponible en: http://tesis.ipn.mx/bitstream/handle/123456789/1 113/yamile\%20ibarra.pdf?sequence=1

Kotler, P. (2000). Introducción al marketing. Madrid: Prentice Hall.
Laguado, F. J. O., Montoya, H. J. C., Pabón, M. K. B., Méndez, C. S., \& Zambrano, J. R. (2013). Transferencias y Desempeño de las Universidades Públicas en Colombia 2010-2011. FACE: Revista de la Facultad de Ciencias Económicas y Empresariales, 12, 41-58.

Mosquera, J. T., \& Reyes, C. M. C. (2014). La valoración y recuperación de saberes como instrumento para la construcción de territorialidad y el desarrollo endógeno. FACE: Revista de la Facultad de Ciencias Económicas y Empresariales, 13(1), 151-170.

Parasuraman, A., Zeithaml, V., y Berry, L. (1985). Un Modelo Conceptual de la Calidad de Servicio. Revista de Marketing, Nro 49.

SampieriHernández, R. y Collado Fernández, C. (2006) Metodología de la investigación. Cuarta Edición. Editorial McGraw Hill.

Santiago Merino, José María (2003) La calidad de servicio bancario: entre la fidelidad y la ruptura. [Tesis Doctoral]. Universidad Complutense de Madrid. Disponible en: http://eprints.ucm.es/tesis/19972000/S/2/S20345 $\underline{01 . p d f}$

Scheaffer, Mendenhall y Ott (1990). Elementos de Muestreo. Grupo editorial Iberoamerica. Mexico D.F.

Serna, H. (2006). Conceptos básicos. En Servicio al cliente. Editorial Panamericana. Bogotá. Colombia.

Stoner, J. y Finch, A. (1996). Administración. $6^{\circ}$ Edición/1996 Prentice Hall

Zeithaml, V y Bitner, M. (2002). Marketing de Servicios: Un enfoque de integración del cliente en la empresa. $2^{\mathrm{a}}$ ed. McGraw-Hill. México. 